\title{
A Novel Gripper Design For Multi Hand Tools Grasping Under Tight Clearance Constraints and External Torque Effect
}

\author{
Mohammad Shaqura \\ Physical Science and Engineering, \\ Mechanical Engineering \\ King Abdullah University of Science and Technology \\ Thuwal, Jeddah 23599, Saudi Arabia \\ Email: mohammad.shaqura@kaust.edu.sa
}

\author{
Jeff S. Shamma \\ Computer, Electrical and Mathematical \\ Science \& Engineering, Electrical Engineering \\ King Abdullah University of Science and Technology \\ Thuwal, Jeddah 23599, Saudi Arabia \\ Email: jeff.shamma@kaust.edu.sa
}

\begin{abstract}
A robotic multi tool gripper design and implementation is presented in this paper. The proposed design targets applications where an actuation task is performed using a wide selection of standard hand tools. The manipulation motion is assumed to be rotational which requires a firm grip to account for external torque on the grasped tool. The setup is assumed to be a conventional workshop panel with hand tools being hanged close to each other, which constraints lateral clearance around the target, and near the wall of the panel, which constraints the depth clearance. Off the shelf grippers are mostly heavy and bulky which make them unsuitable for these requirements. Moreover, they are not optimized in terms of power consumption, simplicity and compactness. These generic grippers are mostly designed for pick and place tasks where no external torques other than those caused by the object weight affects the gripper. The design challenge involves building a gripper that is capable of operating in limited clearance space, firmly grip a variety of standard hand tools with different sizes and shapes. The proposed design is optimized for these objectives and offers a low cost and power consumption solution. The design has been validated in lab and outdoor experiments and has been deployed in real operating platform used in an international robotics competition.
\end{abstract}

Index Terms - Robotic gripper, Autonomous manipulation, Self adjusting mechanism, Adaptive design.

\section{INTRODUCTION}

Robotic grippers have been an active research field over the last decades due to their vast applications in industrial robotics, medical fields, remote exploration, humanoid robotics research and several other areas. Tens of robotic manipulators can be found in a single assembly line in today's modern factory. Those custom designed robots are utilized to perform tasks that are difficult for human workers because it can involve working with huge parts or hazardous tasks. Efficiency and manufacturing consistency are main incentives for deploying industrial automation systems.

Robotic manipulators are characterized by the number of degrees of freedom (DOF), operating space and maximum payload in addition to some other power and software system requirements. An essential part of the manipulator is the end effector element which can be embedded into the manipulator design itself or externally attached for more generic designs. For applications that involve predefined tasks such as welding or painting in factories assembly lines, the first type is usually used. Missions that require higher modularity utilizes manipulators where end effector element can be exchanged.

Human hands offer high resilient and multitasking, for instance grasping objects with different geometries, surface friction and weight distribution. In [1], Sintov presented a gripper design process for picking and placing objects of different geometry by proposing an algorithm that analyze the geometry of an object and build a library of best grasping points utilizing a 3-fingered robotic gripper. Multi-finger grippers can simulate human grasping especially if they are coupled with force/torque feedback [5] or tactile accurate sensing [6]. However, this usually comes associated with the cost of controlling higher number of actuators and sensor data processing which increase the size and weight and make the control task more challenging.

Other designs targets specific operational requirements rather than mimicking the structure of human hands. These designs prove effectiveness for their target tasks like object picking [15], [16]. A two plates intelligent design is presented in [7] for picking objects with simple geometry. A magnetic gripper design and development with the assumption of metal objects lifting and unstructured environment is presented in [2]. Mechanically adaptive two sided robotic gripper designs are presented in [9] and [12] where the concept of compliance is utilized in the design to account for different geometries and secured grip of objects. A novel design to grip fragile or soft objects is developed in [11] by deploying deformable fingertips filled with liquid-gel material. A bio-inspired gripper based on inflatable micro-fiber contact surface is presented in [14]. The gripping mechanism attached to the object by controlling the pressure of the air going flowing to the contact surface.

Soft robotics is an evolving research field with the advancement of material science and algorithms. Flexibility and compliance are important features of future robotic gripper designs. A low cost flexible design is presented in [3]. A 
design that is based on artificial muscle is discussed in [4] and a hydraulic/pneumatic driven claw like gripper is presented in [8]. The advancement in the technology of 3D printing allows rapid prototyping of low cost grippers [10], [13].

Our goal is to design a robotic gripper to grasp hand-tools available in a conventional workshop, such as, wrenches or other tools that fits vertically in the gripping space. The target grasp position is the tool nick allowing manipulation of an external system, e.g. a valve. The operating clearance is limited around the tool and a firm grip is required as the picked tool is used to perform a manipulation task that will exert back torque on the tool. Next, we present the design requirement. In section (III), the proposed gripper design is discussed and the full gripper system is presented in section (IV). Then experimental validation and gripper in actual operation is presented before concluding and presenting future directions of this work.

\section{DESIGN REQUiREMENTS}

The main design objective is to build a robotic gripper for grasping hand tools that are typically hanged on a workshop panel, perform a manipulation task and place tool back autonomously. The system is assumed to have the capability to use standard hand-held tools such as wrenches where the effective elements are on one or both ends.

An example of experimental setup is shown in figure (1) where a set of 12 standard wrenches are hanged to a workshop wooden panel. The keys attachment pins are $5 \mathrm{~cm}$ apart which leaves a tool clearance margin of only $2.5 \mathrm{~cm}$ from the center of each key. Grippers with horizontal axis closure mechanisms or multi fingered bulky grippers are not suitable for this task as collision with neighboring tools can occur. The depth clearance is another design parameters that can be challenging for most of off-the-shelf robotic grippers. We mean by that, the maximum distance between the gripped object and the wall which is reflected in this scenario by the holding pin offset from the wall of the panel, $15 \mathrm{~mm}$.

The picked tool is needed to be maintained in the same default orientation or slightly off with small margin with respect to the robotic manipulator Cartesian coordinate system. The tool need to be aligned with the vertical axis while maintaining attitude Euler angles offset. The tool is required to be firmly gripped to maintain its orientation while performing a manipulation task, for example, opening or closing a valve. Side grasping is not optimal in this scenario where the contact surface is minimal and slip or complete fall of the tool can occur especially when high torque acts on the tool. Accurate alignment of the tool simplifies the control task which prevents performing complex trajectories by the robotic manipulator which requires computational resources and accurate force and computer vision feedback. The grip should also be immune against external forces and torques in all directions.

Variations in geometry and dimension need to be addressed so the designed gripper has the capability to grasp a range of tools sizes. For the set of wrenches example, the tools vary in size between 10 and $24 \mathrm{~mm}$ as shown in table (I). The range of tool heights varies between 140 and $280 \mathrm{~mm}$

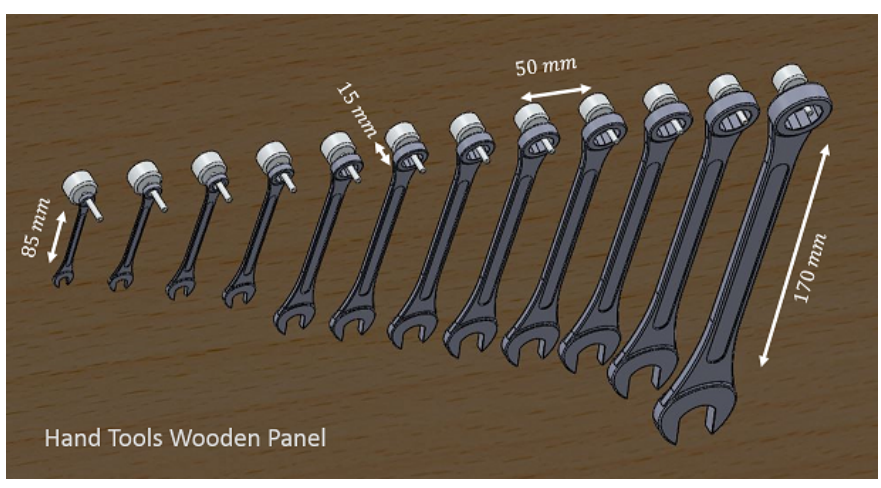

Fig. 1. Tilted view of wrench tools panel example with keys varying from size 10 to 24 , showing the horizontal and depth clearance constraints

TABLE I

STANDARD SPANNER KEYS DIMENSIONS $(\mathrm{mm})$

\begin{tabular}{|c||c|c|c|c|}
\hline Key Size & Full Height & Nick Height & Width & Thickness \\
\hline 10 & 140 & 85 & 12.5 & 4.5 \\
\hline 15 & 190 & 115 & 17.5 & 6 \\
\hline 19 & 230 & 140 & 23 & 7.5 \\
\hline 24 & 280 & 170 & 27.5 & 9 \\
\hline
\end{tabular}

but this does not represent the effective heights of the tools which is the maximum length of contact between the key and the gripper excluding both operating ends. We call this the Nick Height and it varies between 85 and $170 \mathrm{~mm}$ for the wrenches in consideration. The width of the tool nick and the thickness vary between $12.5-27.5$ and 4.5-9 $\mathrm{mm}$ respectively. This variability poses design challenges as the picked tool for manipulation is not known in advance. The designed gripper should be able to grasp a tool of size 10 and 24 with the same performance and reliability.

Further design specifications include minimizing the number of degrees of freedom which simplifies the operation logic and limit power consumption. The gripped tool is required to actuate a valve with nominal torque of $5 \mathrm{~N} . \mathrm{m}$. No assumption of ferrous tools or flat surface finish is assumed which makes the use of magnetic or suction cup grippers not ideal for this application. We aim to design a low cost, light weight and easy to implement and operate robotic gripper autonomously as part of bigger systems with high efficiency and reliability while meeting all design requirements.

\section{GRIPPER DESIGN AND ANALYSIS}

In this section, a gripping mechanism that is simple and effective is presented where we address each of the design requirements mentioned in the last section.

\section{A. Robot Coordinate System}

The following Cartesian coordinate system is assumed which matches the coordinates of the robotic arm where gripper is mounted. The origin coincides with the robotic arm tip point when the robot is in some default ready position $\left(x_{0}, y_{0}, z_{0}\right)$. The $x$ and $y$ axes are aligned with the global horizontal and vertical translation of the robotic arm and the 
$z$-axis is pointing out of the gripper tip point. In ready position, it is assumed that the last joint of the arm is aligned with the $z$ axis. The gripper coordinates is similar to the arm with shift in the origin. The end effector origin $\left(x_{g}, y_{g}, z_{g}\right)$ is given by:

$$
\begin{aligned}
& x_{g}=x_{0}, \\
& y_{g}=y_{0}+h+\alpha, \\
& z_{g}=z_{0}+L-\beta-\gamma
\end{aligned}
$$

where $h$ is the vertical displacement between the center of the robotic arm and the gripper mount. Choosing $h$ depends on the robot capability and, manipulation task, tool length and control strategy. $\alpha$ is the distance in $y$ direction between the edge and the center of the gripper. This parameter depends on the geometry and orientation of the tools to be grasped. Table (I) for instance shows the effective contact area for tools of different sizes. In the wrench example, the gripper need to effectively grasp tools of lengths $85-170 \mathrm{~mm}$. $L$ is the full gripper length which is minimized while prioritizing minimum width due to clearance constraints along the $x$ axis. $\beta$ is the maximum offset between the gripper front edge and the effective grasping region. It is chosen based on the clearance constraint between the tool and the wall as seen in figure (1). $\gamma$ is the radius of the biggest circle that can fit inside the grasp region. The size of this circle is another design parameter, the bigger the circle, the bigger the tool width and thickness it can accommodate and the wider the gripper overall width which is undesirable according to the requirements.

\section{B. Grasp Mechanism}

The robotic gripper design shown in figure (2) operates using two actuators acting on two geared mechanisms. Multiple design iterations and refinement have been performed, the current design is easy to integrate, low building cost and meet the requirements in section II. The gripper is designed and studied using Solidworks engineering software and its motion analysis toolbox [17].

The gripper mechanism is inspired by human hand motion when grasping a tool for manipulation task. For a tool that is hanged on a panel, the way we grasp it differs from one person to another but the majority follows similar strategy. Initially, one need to make sure that there is sufficient contact area between the hand and the tool. Then, the fingers push the tool against the forehand and secure it before performing the manipulation task. In a similar way, the first actuator produces a rotational motion around an axis that is parallel to the $y$-axis. This motion move a geared cylindrical shell part to close the circular grasp region and that introduces the needed contact surface with the tool. The second actuator acts on a linear gear connected to a curved plate that pushes the tool against the cylindrical wall so it is jammed. Figure (2) shows the gripper while a key is grasped, the actuator motion directions are indicated by the red arrows. The open and close states are shown in figure (3). The gripping force acts directly in the $z$ direction of the gripper. Under the assumption that the gripper is aligned with the last joint of the robotic arm, the force also acts in the $z$-axis of the manipulator Cartesian coordinate

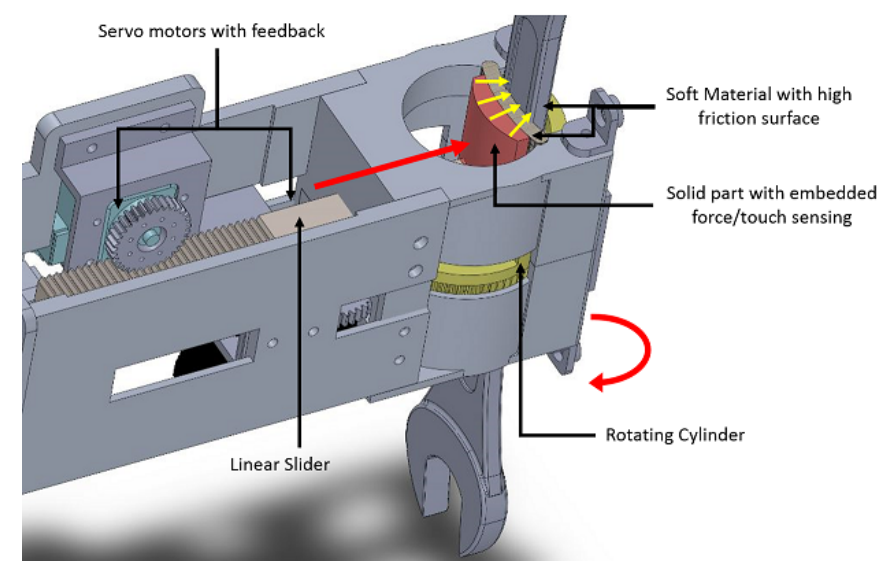

Fig. 2. Gripper 2 DOF Mechanism and Feedback
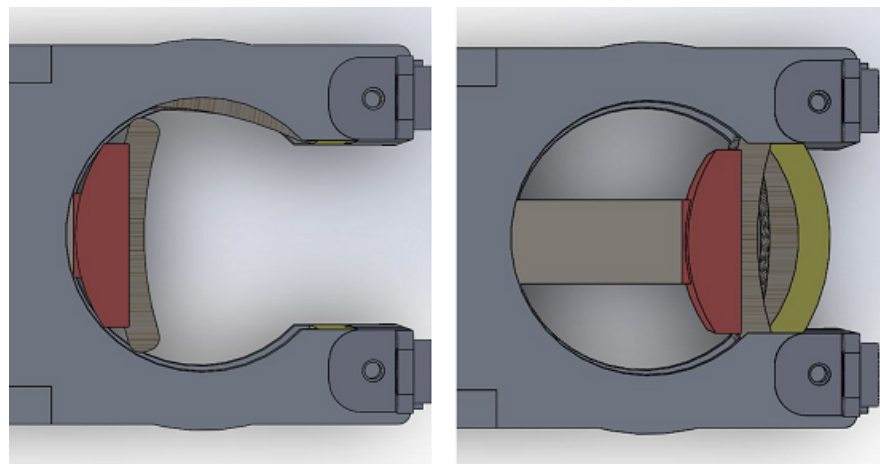

Fig. 3. The two gripper states, left: Open/Idle and right: Close/Grasp

system which makes the approach control strategy easier. The circular design increases the effective gripping space while limiting the overall width of the gripper which meets the constraints on the horizontal clearance to avoid collision with neighboring tools and maximize accessibility.

The forces and torques acting on the grasped tool can vary depending on the tool approach strategy and tool orientation. The external torques acting on the tool throughout the manipulation process are also affected by the tool orientation with respect to the gripper origin. The maximum force occur when the gripper element of action (red colored object in figures $(2,3)$ is in full alignment and contact with the key. This happens when the pitch, $\theta$ and roll $\phi$ angles are close to zero. The applied grip force is affected by the shift in attitude:

$$
F_{\theta}=F \cos (\theta) \quad, \quad F_{\phi}=F \cos (\phi)
$$

The proposed design eliminates the need for perfect alignment with the tool which makes it less sensitive to attitude offset initially. The mechanism account for this deviation without considering complex approach algorithms and feedback systems with high accuracy.

The offset in the key yaw angle $\psi$ affect the manipulation control strategy. Ideally, the tool acting element should be aligned with the $y$-axis which means minimum deviation of the yaw angle. For the wrench example, the lateral force acting 
TABLE II

Mechanical and Power Specifications

\begin{tabular}{|c||c|}
\hline Horizontal Span & $60 \mathrm{~mm}$ \\
\hline Gripping Area & $40 \times 30 \mathrm{~mm}$ \\
\hline Front Tip Offset & $10 \mathrm{~mm}$ \\
\hline Minimum Object Thickness & $2.5 \mathrm{~mm}$ \\
\hline Maximum Object Width & $32 \mathrm{~mm}$ \\
\hline Idle Power Consumption & $2.4 \mathrm{~W}$ \\
\hline Active Power Consumption & $19.5 \mathrm{~W}$ \\
\hline Grip Force & $115 \mathrm{~N}$ \\
\hline Lateral Friction* & $45 \mathrm{~N}$ \\
\hline
\end{tabular}

* Friction force depends on object material and surface finish, stainless steel tools are assumed in the calculations.

on the center point of grip due to applied manipulation torque is given by:

$$
F_{c} \approx \frac{T_{e x t}}{h+\frac{L}{2}}
$$

To limit the effect of external torque $T_{\text {ext }}$ on the key orientation, a firm grip is desired. This is accomplished by increasing the jamming force along the $z$-axis and increase the lateral friction force of the contact interface of the tool and the gripper surfaces. For a wrench, the front and back faces forms the largest portions of the total gripping surface. When the gripper is in closing transition, two surfaces with very small curvature are in physical contact with the tool. These two surfaces are covered with two layered material that helps in the grasp task. The first layer is a simulated sponge that provide the properties of softness to reduce the impact on the actuator in case of sudden change of torque and resilience to retain its form under variable applied forces. This layer allows jamming the gripped tool in a full 360 envelop covering all the tool sides which contributes to securing the grip further. The second layer of the covering material which is in direct contact with the tool surface is made of silicon carbide coating ( $\mathrm{SiC})$ that provides high surface friction between the gripper and the tool surfaces. Table (II) shows geometrical, power rating and force specifications of the current deployed version of the gripper. The circular design that is based on containment by the cylindrical part before applying the grip force helps in meeting the depth constraint for grasping the tool. The current design allows to grasp the tool with up to $10 \mathrm{~mm}$ of clearance between the tool and the panel.

The contact surface of the sliding part of the mechanism is equipped with touch sensing that indicates engagement with the tool which helps in autonomous operations. The unique feature of this design is that only one actuator is continuously active while the gripper is closed. The actuator controlling the cylindrical body is only active for few seconds after reaching the gripping position, when the tool is inside the grasping region. This actuator goes to idle state until an open command is initiated. This reduces the overall power consumption by operating with one actuator while maintaining a firm grip, table (II). The motor load feedback allows variable grip force based on the external torque feedback measured by the robotic arm 6-axes force/torque sensor. The current drawn by the actuator can be reduced or increased as needed which further contribute to limit power consumption.

\section{RISC G1 RobotiC GRIPPER SYSTEM}

In the previous section, the gripper mechanism is introduced with the focus on mechanical operation. In this section, we introduce the robotic gripper as a smart modular system with mechanical, sensing and vision capabilities and how this system is integrated with other robotics systems.

The main components of the robotic gripper system consists of the following modules:

- Grasp Mechanism: this is the core part of the gripper which consists of the mechanical subsystem. It includes two smart servo motors or normal servos and rotational and linear geared parts in addition to the special gripping material. It is discussed in details in section (III).

- Feedback Module: it consists of 3 main components, inertial measurement, servo feedback and tactile sensing. The gripper is equipped with an electronic 9-axis IMU (accelerometer, gyroscope and magnetometer) and Extended Kalman Filter (EKF) estimator of euler angles. This is a direct embedded attitude measurement. Two servo related feedback exist, current servo position and load. The current position is an indication of object size or tool thickness in addition to transition states. The load feedback is an indicator of the grip force which is useful for varying force grip adapting with the external torque effects. Tactile sensing is embedded in two locations. The first touch sensing is embedded in the part attached to the linear gear that jams the tool. It is an indicator of the tool touch and engagement. The second form of tactile sensing is embedded in the corners of the gripper edge as shown in figure (4). These are not utilized for grasping mechanism, rather they are used for accurate alignment of the gripper with the panel.

- Vision Module: two serial cameras are embedded for vision feedback. The first camera is used for tool/valve detection and classification. The second camera is used for tool alignment with respect to the gripper and robotic arm in addition to valve sizing.

- Hardware Interface: the full robotics system run on a central computer where all subsystems, including the gripper system, communicate. The interface with the main CPU is done using serial connection. All hardware components are connected to a microcontroller module with various needed interfaces such as, digital and analog I/O, I2C, USB and RS485.

- Software Interface: it consists of two layers, hardware layer and system layer. The first layer is implemented locally on the microcontroller for the purpose of reading signals coming from the hardware devices and writing data to output devices. It acts as a bridge between the system layer and hardware by processing the data from sensors to meaningful feedback and convert the control signals to hardware commands. The system layer is built 


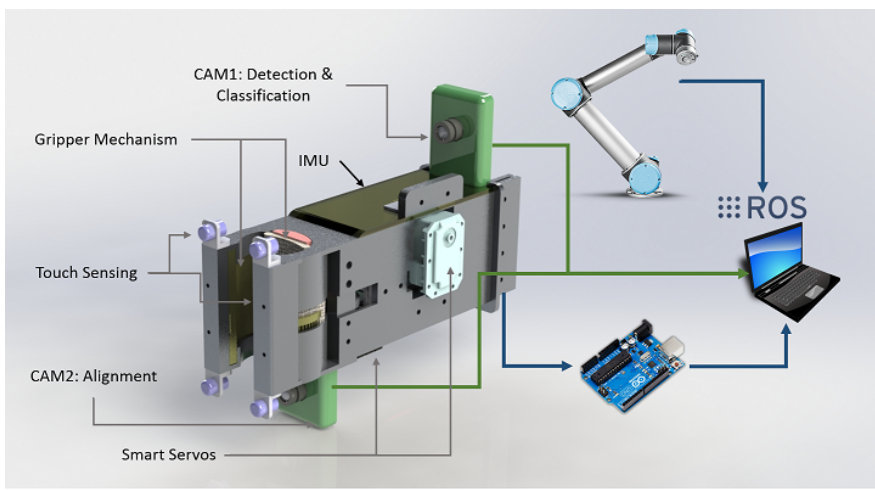

Fig. 4. Robotic Gripper System Integration

utilizing the Robotics Operating Systems (ROS) tools [18] which simplifies the integration with other subsystems (robotic manipulator, sensors, mobile robots) due to its modular nature.

The full gripper system and integration with the main CPU and the robotic arm is shown in figure (4). The control algorithm is developed using ROS where all hardware modules including the gripper are integrated. This introduces flexibility in the system and make addition or removal of devices (other types of servos) or sensors (sonars or laser range finders) easier. The gripper operates in three different modes which are:

- Grasp Mode: it is the main operation mode of the gripper and it takes one of two states, open/idle and closed as shown in figure (3). Manipulation is part of this mode whether constant or varying grip force is applied.

- Sizing Mode: this is performed by the front actuator that move the cylindrical part. Unlike Grasp mode, where the goal is to close the grasping region and introduce the back contact surface with the tool. This moving part in this mode is used for object sizing, mechanical mean of valve sizing for instance. The servo position and load feedback are used to indicate if an object is jammed between the gripper fixed wall and the edge of the rotating disc.

- Alignment Mode: the embedded four corners points tactile sensing are the main feedback elements in this mode. The purpose of this mode is to align the gripper with the panel with high accuracy before starting the main operation. The robotic arm move slowly toward the panel until any touch feedback is detected. The robotic arm joints are controlled to adjust the gripper orientation such that all four touch sensors are toggled simultaneously at the moment the gripper touches the panel.

The manipulation process starts with the alignment model then grasp mode. Sizing mode is an optional utility if computer vision feedback does not detect valve size accurately.

\section{EXPERIMENTAL AND OPERATIONAL VALIDATION}

The gripper is mounted to one of the mobile robotic manipulator platforms in the Robotics, Intelligent Systems and Control (RISC) lab at King Abdullah University of Science and Technology (KAUST). The robotic manipulator used for the experiment is Universal Robot, UR5 and the ground vehicle platform is ClearParth Robotics, Husky UGV. The platform is equipped with a central mini-ITX CPU and a number of devices and sensors such as: inertial measurement unit (IMU), wide view camera, lidar and force/torque sensor.

The experimental setup is based on an autonomous task scenario that is part of an international robotics challenge, $M o$ hamed Bin Zayed International Robotics Challenge (MBZIRC 2017). The challenge requires a UGV platform to search for a panel that has a number of attached tools and a valve that needs to be actuated. The UGV need to be equipped with a robotic arm manipulator, computation module, sensing and a robotic gripper to complete the task autonomously. The task is to find the panel, indicate the size of the valve, detect the suitable tool size, pick the right tool and close the valve.

The robotic UGV scans a field for the target panel. When the panel is found, the UGV circles around it until the face with the attached wrenches and valve is detected. The platform aligns itself with the panel and the robotic manipulator moves to the ready state where its tip points toward the panel and this position defines the initial state for manipulation phase. The first step is panel alignment to accounts for inaccuracies in proximity measurements. This is done with the help of the four corner touch sensors embedded in the gripper body. Alignment of the gripper front face and the panel within a millimeter accuracy can be achieved by iteratively adding small adjustment to the manipulator joints. Additionally, accurate distance from the panel can be found. The pitch and roll angles are adjusted with this mechanism with the help of the last two robotic arm joints. The tool is initially pointing downward under the assumption of flat area where the robot and the panel stand. Once the alignment process is completed, a virtual origin and robotic arm/gripper navigation axes are established.

The size of the valve is detected using computer vision algorithms utilizing a wide dynamic range camera. Then, the robotic manipulator navigates to the correct key that is also detected using vision feedback. The gripper closing transition starts when the correct key is inside the grasp region. The first motor rotate the cylinder to confine the key prior to initiating the gripping actuator. The second actuator controls a linear gear that push the wrench nick against the other surface and jam it with the help of the soft material and the high friction layer that is in contact with the tool. Once the tool is grasped, the robotic arm autonomously navigates to the valve location and place the key on the valve to start the 360 degrees rotational manipulation. The tool-valve engagement should be maintained to complete the rotation which indicates task completion. Figure (5) shows an autonomous valve engagement and actuation experiment performed at KAUST stadium. Figure (6) shows real-time gripper operation while grasping a wrench in MBZIRC robotic competition held in Abu Dhabi, UAE, 2017. Note that some minor modifications on the gripper system discussed in the last section is done for different experiments, e.g. location of the cameras, type of cameras, type of motors or gripper mounting. 


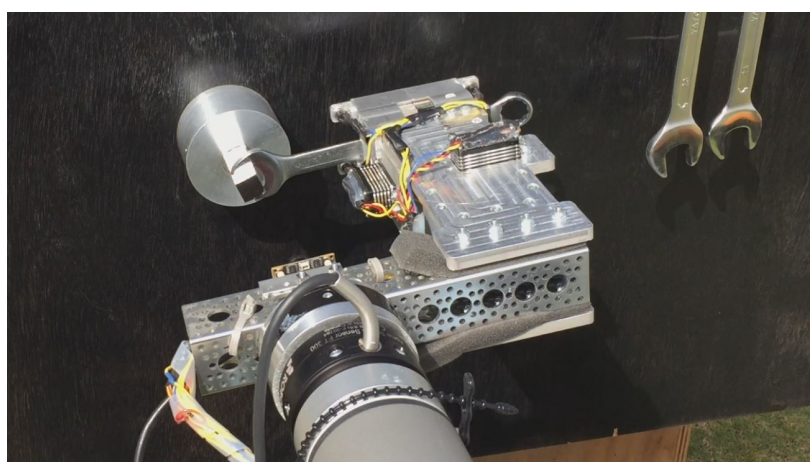

Fig. 5. Autonomous valve engagement and rotation using a gripped tool

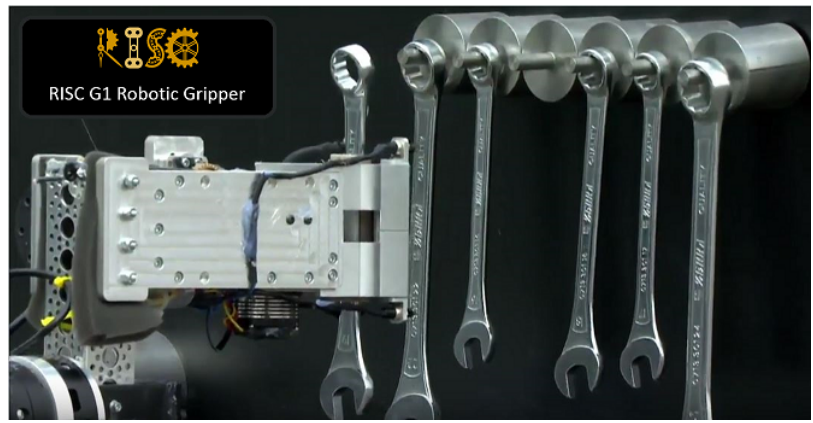

Fig. 6. RISC G1 gripper tool picking during MBZIRC trial

\section{CONCLUSION}

A robotic gripper design for multi tool sizes that operates in a limited clearance space is presented. The grasped tool is used to perform a manipulation task where external toques act on the tool. The designed gripper mechanically handles most of the operation requirements which reduce complexity of controlling the robotic arm and make the system less sensitive to measurements uncertainties. The gripper software system is integrated with the robotic arm and other devices in the system through the open source robotics operating system, (ROS) running on a central computer. The designed gripper is validated through lab experiments and outdoor testing. It is also deployed in operational robotic platform used in an international robotics competition. Future directions include improvement in the hardware and software subsystems. Adding flexibility to the mechanism can improve reliability by introducing compliance to the system. Different gripping materials will be tested to improve grip force and minimize the risk of slip. Grasping other similar tools will be studied and geometrically analyzed. Variable grip force based on force/torque feedback or computer vision estimates will be investigated. Several improvements will be added to the gripper ROS packages and nodes to improve performance, modularity and integrity with other robotics systems.

\section{ACKNOWLEDGMENT}

The authors would like to thank Dr. Samet Guler (Postdoctoral fellow, KAUST) and Mr. Mohammed AlGarni (PhD student, KAUST) for their valuable feedback throughout the design and implementation of the presented work.

\section{REFERENCES}

[1] A. Sintov, R. Menassa and A. Shapiro, "A gripper design algorithm for grasping a set of parts in manufacturing lines". Mechanism and Machine Theory 105, pp. 1-30, 2016.

[2] R. Debanik, "Development of novel magnetic grippers for use in unstructured robotic workspace". Robotics and Computer-Integrated Manufacturing 35, pp. 16-41, 2015.

[3] P. Dalibor, S. Jovic, O. Anicic, B. Nedic, and B. Pejovic, "Analyzing of flexible gripper by computational intelligence approach". Mechatronics 40, pp. 1-16, 2016.

[4] E. Hamburg, V. Vunder, U. Johanson, F. Kaasik, and A. Aabloo, "Soft shape-adaptive gripping device made from artificial muscle". In SPIE Smart Structures and Materials Nondestructive Evaluation and Health Monitoring, pp. 97981Q-97981Q. International Society for Optics and Photonics, 2016.

[5] C. Seguna and M. Saliba, "The mechanical and control system design of a dexterous robotic gripper". The 8th IEEE International Conference on Electronics, Circuits and Systems, vol. 3, pp. 1195-1201. IEEE, 2001.

[6] P. Angelika, S. Einenkel and M. Buss, "Multi-fingered telemanipulationmapping of a human hand to a three finger gripper". The 17th IEEE International Symposium on Robot and Human Interactive Communication, pp. 465-470. IEEE, 2008.

[7] A. Zaki, A. Soliman, O. Mahgoub and A. El-Shafei, "Design and implementation of efficient intelligent robotic gripper". The 2010 International Conference on Modelling, Identification and Control (ICMIC), pp. 710716. IEEE, 2010

[8] U. Ganesha, P. Sreedharan and K. Aditya, "Robotic gripper driven by flexible microactuator based on an innovative technique". IEEE Workshop on Advanced Robotics and its Social Impacts (ARSO), pp. 111-116. IEEE, 2010.

[9] V. Timothy, H. Staab, S. Breisch, S. Soetebier, T. Stahl, A. Hackbarth and S. Kock, "A flexible robotic gripper for automation of assembly tasks: A technology study on a gripper for operation in shared human environments". IEEE International Symposium on Assembly and Manufacturing (ISAM), pp. 1-6. IEEE, 2011.

[10] R. Raymond, L. Odhner and A. Dollar, "A modular, open-source 3d printed underactuated hand". IEEE International Conference on Robotics and Automation (ICRA), pp. 2737-2743. IEEE, 2013.

[11] M. Ryoji, T. Watanabe and M. Uchida, "Delicate grasping by robotic gripper with incompressible fluid-based deformable fingertips". IEEE/RSJ International Conference on Intelligent Robots and Systems (IROS), pp. 5469-5474. IEEE, 2013.

[12] C. Fei, F. Cannella, C. Canali, T. Hauptman, G. Sofia and D. Caldwell, "In-hand precise twisting and positioning by a novel dexterous robotic gripper for industrial high-speed assembly". IEEE International Conference on Robotics and Automation (ICRA), pp. 270-275. IEEE, 2014.

[13] T. Yedige, K. Telegenov and A. Shintemirov, "An open-source 3D printed underactuated robotic gripper". IEEE/ASME 10th International Conference on Mechatronic and Embedded Systems and Applications (MESA), pp. 1-6. IEEE, 2014.

[14] S.Sukho, C.Majidi and M.Sitti, "Geckogripper: A soft, inflatable robotic gripper using gecko-inspired elastomer micro-fiber adhesives". IEEE/RSJ International Conference on Intelligent Robots and Systems (IROS 2014), pp. 4624-4629. IEEE, 2014.

[15] N. Correll, K. Bekris, D. Berenson, O. Brock, A. Causo, K. Hauser, K. Okada, A. Rodriguez, J. Romano, and P. Wurman, "Lessons from the amazon picking challenge". arXiv preprint arXiv:1601.05484, 2016.

[16] C. Eppner, S. Hfer, R. Jonschkowski, R. Martn-Martn, A. Sieverling, V. Wall and O. Brock, "Lessons from the amazon picking challenge: Four aspects of building robotic systems". Proceedings of Robotics: Science and Systems, AnnArbor, Michigan, 2016.

[17] P. Kurowski, "Engineering Analysis with SolidWorks Simulation 2013". SDC publications, 2013.

[18] M. Quigley, K. Conley, B. Gerkey, J. Faust, T. Foote, J. Leibs, R. Wheeler, and A. Y. Ng. "ROS: an open-source Robot Operating System". In ICRA workshop on open source software, vol. 3, no. 3.2, p. 5.2009. 